\title{
Narración frente al neoliberalismo en la formación docente. Visibilizar para transformar
}

\section{Resumen}

Este artículo de reflexión se deriva de la investigación "La construcción de la identidad profesional del estudiantado del grado de Primaria". Pretendo hacer una revisión del modo en el que la investigación narrativa contribuye a la construcción de la práctica docente, a partir de la revisión del relato escolar que los profesionales de la enseñanza elaboran en el contexto de la moral neoliberal. Pongo de manifiesto cómo el carácter contingente e histórico de la construcción de la realidad, por medio del relato que hacemos de ella, cuestiona la ideología neoliberal dominante en la formación y la práctica docente.

\section{Palabras clave descriptor}

Política educacional, análisis cualitativo, formación de docentes de Primaria, docente de escuela primaria.

\section{Transferencia a la práctica}

Este trabajo tiene una especial incidencia en la reconfiguración de los modos como se forma al profesorado en la actualidad. Se intenta reconducir la moral neoliberal subyacente en las prácticas actuales, mediante la construcción de otro relato de escuela en un nuevo modelo de formación. En un contexto de fuertes reformas en la universidad y en los sistemas escolares, entiendo que es imprescindible visibilizar la ideología presente en estas propuestas. El relato escolar de los participantes se antoja un privilegiado punto de partida para hacer posible una práctica crítica y reflexiva de formación.

Para citar este artículo / To cite this article / Pour citer cet article / Para citar este artigo

Rivas-Flores, J. I. (2014). Narración frente al neoliberalismo en la formación docente. Visibilizar para transformar. magis, Revista Internacional de Investigación en Educación, 7(14), 99-112. 


\section{Key words plus}

Educational Policy, Qualitative Analysis, Primary Teacher Training, Primary

School Teacher.

\section{Abstract}

This reflective paper stems from the research entitled "The Construction of the Professional Identity of Elementary School Students". I intend to review the way in which narrative research is contributing to the construction of teaching practice, based on the review of the school narrative that teaching professionals are producing in the context of neoliberal moral. The paper highlights how the contingent and historical character of the construction of reality, through the story we make out of it, questions the dominant neoliberal ideology in training and teaching practice.

\section{Transference to practice}

This work has a particular impact in the reconfiguration of the ways teachers are being educated nowadays. It tries to redirect the neoliberal moral that underlies the current practice, through the construction of another school narrative of a new training model. In a context of strong reforms in both the university and school systems, I understand that it is essential to render visible the ideology present in these proposals. For the school, narrative of participants represents a prime opportunity that enables a critical practice and a reflective training.

\section{Mots clés descripteur}

Politique éducationnelle, analyse qualitative, formation d'enseignants de l'école primaire, enseignant de l'école primaire.

\section{Résumé}

Cet article de réflexion dérive de la recherche "La construction de l'identité professionnelle des élèves d'éducation primaire». On vise faire un bilan du mode dont la recherche narrative est en train de contribuer avec la construction de la pratique de l'enseignant, à partir de la révision du récit scolaire que les professionnelles de l'enseignement sont en train d'élaborer dans le contexte de la morale néolibérale. Cela évidence le caractère contingent et historique de la construction de la réalité, au moyen du récit que nous en faisons, et on question l'idéologie néolibérale dominante dans la formation et la pratique de l'enseignant.

\section{Transfert à la pratique}

Ce travail a une incidence spéciale dans le façonnement des modes dont on forme aux enseignants dans l'actualité. On essaye de reconduire la morale néolibérale sous-jacente dans les pratiques actuelles, au moyen de la construction d'autre récit d'école dans un nouveau modèle de formation. Dans un contexte de fortes réformes dans l'université et dans les systèmes scolaires, on comprend qu'il est indispensable de rendre visible l'idéologie présente dans ces propositions. Le récit scolaire des pratiquantes est un privilège et un point de départ pour donner la possibilité de faire une pratique critique et réflexive de la formation.
Palavras-chave descritor

Política educacional, análise qualitativa, formação de docentes de escola primária.

\section{Resumo}

Este artigo de reflexão deriva-se da pesquisa "A construção da identidade profissional do docente do grau primário". Pretendo fazer uma revisão do modo em que a pesquisa narrativa está contribuindo à construção da prática docente, a partir da revisão do relato escolar que os profissionais do ensino estão elaborando no contexto da moral neoliberal. Destaca-se como o caráter contingente e histórico da construção da realidade, através do relato que fazemos dela, questiona a ideologia neoliberal dominante na formação e na prática docente.

\section{Transferência à prática}

Este trabalho tem uma especial incidência na reconfiguração dos modos em que se forma o professorado na atualidade. Tenta-se reconduzir a moral neoliberal subjacente nas práticas atuais, através da construção de outro relato de escola em um novo modelo de formação. Em um contexto de fortes reformas da universidade e dos sistemas escolares, entendo que se torna imprescindível fazer visível a ideologia presente a estas propostas. O relato escolar dos participantes necessita de um privilegiado ponto de partida para tornar possível uma prática crítica e reflexiva de formação. 


\section{Introducción}

La investigación narrativa y biográfica no busca descubrir la realidad que se nos presenta, sino comprender el relato construido social e históricamente para facilitar su transformación. Desde esta premisa afronto este artículo, en el que intento evidenciar cómo este tipo de investigación puede ser parte de las dinámicas de cambio y transformación social en el marco neoliberal dominante. Entiendo que el contexto actual está construyendo una realidad a partir de la hegemonía de lo que denomino "moral neoliberal" en las prácticas escolares.

Mi reflexión transita por uno de los ejes sobre los que gravita la diferencia de lógica entre la investigación interpretativa y la positivista: el sentido de la realidad y la relación con los sujetos, tanto investigados como investigadores. Frente a la posición naif del positivismo, que considera que la misión del investigador es desvelar la realidad desde una supuesta independencia entre ellos, la investigación interpretativa y la biográfica narrativa, en particular, entienden que existe una tensión crítica entre ambos, en una dinámica de reconstrucción mutua: el sujeto construye la realidad a partir del relato que hace de ella, al tiempo que este mismo relato orienta la construcción del propio sujeto.

En este artículo analizo esta tensión crítica desde el trabajo de investigación que estoy efectuando en las Universidades de Málaga y Almería, junto con la Universidad de Jaén. A efectos de este texto, manejaré los datos de la Universidad de Málaga, ya que son los que hasta el momento he trabajado de forma personal. Esta investigación plantea una revisión del actual modelo de formación del profesorado, con base en el estudio de "la construcción de la identidad profesional en los estudiantes del grado de Primaria". Voy a centrarme en el profesorado de reciente incorporación a la profesión y en los estudiantes del grado de Primaria. Parto del principio de que la socialización profesional docente es un proceso complejo que se elabora a partir de la construcción que los sujetos van haciendo sobre qué es la escuela y cómo funciona y de sus experiencias biográficas e históricas tanto en la institución como en los marcos sociales, culturales y políticos en los que funciona. De acuerdo con esto, los sujetos (el alumnado del grado de Primaria y el profesorado novel en ejercicio) elaboran sus relatos escolares, que constituyen el núcleo de la investigación.

En este contexto, la investigación narrativa puede ofrecer herramientas para comprender el proceso de construcción de estos relatos y la forma como "sedimentalmente" van constituyendo el modo de hacer y pensar en la profesión docente. Me preocupa, por tanto, indagar cómo se pueden reelaborar estos relatos, más allá de las formulaciones conceptuales, para provocar una transformación de las prácticas. ¿Es posible pensar un cambio de relato en los marcos culturales y políticos que está construyendo la moral neoliberal? Y, según lo planteado, ¿la investigación narrativa puede representar un factor de cambio en la constitución de las prácticas? ¿De qué modo la formación docente se sitúa en relación con estos relatos? Estas son algunas de las cuestiones que surgen como reflexión crítica acerca de la capacidad de la investigación biográfico-narrativa, para construir una nueva realidad educativa.

Para ello, en primera instancia voy a plantear una revisión conceptual que sitúe el problema que presento desde una revisión de las relaciones entre vida y narrativa. A continuación, muestro los ejes principales de la investigación y algunos resultados relevantes. Por último, analizo la construcción de la práctica escolar que emerge del proceso de elaboración de la identidad profesional a lo largo de la experiencia escolar y del proceso de formación en la universidad.
Descripción del artículo | Article description | Description de l'article | Artigo descrição

Este artículo de reflexión se deriva de la investigación "La construcción de la identidad profesional del estudiantado del grado de Primaria", que busca revisar las oportunidades de la investigación narrativa, para poner en cuestión el discurso neoliberal presente en las prácticas escolares actuales. Se hace una revisión del campo de reflexión en torno a la construcción del relato, su relación con la realidad y los procesos constitutivos de las instituciones escolares. Por otro lado, por medio de la descripción de la investigación se analiza el discurso escolar y las posibilidades de construir un relato de escuela que transforme las prácticas escolares. 
Vida y narrativa: cuestionar la realidad

\begin{abstract}
La vida no es la que uno vivió, sino la que uno recuerda y cómo la recuerda para contarla García-Márquez (2002, p. 7).
\end{abstract}

Nada mejor que iniciar este trabajo con la cita con la que GarcíaMárquez (2002) presenta su libro de memorias. Nadie mejor que un mago de la literatura para manifestar el sentido de la narración como expresión de aquello que podemos llamar realidad. Convencionalmente, se entiende que el relato autobiográfico dice aquello que le ocurrió a un sujeto a lo largo de su vida. Se cree que aquello sucedió en realidad, ya que es el propio sujeto quien lo cuenta. Nos movemos de acuerdo con lo que Lejeune (1994) denominó el pacto autobiográfico, una suerte de contrato entre autor y lector por el cual el primero se compromete a contar la verdad sobre su vida y el segundo a creer el relato que se le ofrece. En sus propias palabras, "el personaje no tiene nombre en la narración, pero el autor declara que coincide explícitamente con el narrador (y por lo tanto con el personaje, ya que es una narración autodiegética')" (p. 68). Este pacto, por tanto, elimina la posibilidad de la ficción, incluso en el caso de que la historia sea falsa, ya que, como expresa este autor, la mentira no deja de ser sino una categoría de la autobiografía, pero nunca puede considerarse ficción.

El problema no se plantea en términos de veracidad de los hechos que se narran, sino de la identificación entre narrador y personaje; por ello, resultan pertinentes los dos filtros que García-Márquez (2002) pone en el relato autobiográfico: el recuerdo y la narración. Me atrevo a afirmar que no existe eso que decimos que el sujeto vivió, sino solo la narración de lo que recuerda. Desde esta óptica, es necesario considerar dos cuestiones que afectan de modo indefectible el proceso de construcción del conocimiento biográfico. Por un lado, el recuerdo es histórico; esto es, se construye desde el conjunto de experiencias vividas y los contextos en donde acontecieron. Por otra parte, el acto de narrar es contingente, fruto de las condiciones en las que se produce. Ambas cuestiones nos remiten a una mirada netamente constructivista y crítica. La vida no es más que la narración que hacemos de ella en unas condiciones particulares.

\title{
La construcción narrativa de la realidad
}

Resulta pertinente retomar la diferenciación que Conle (2014) señala al referirse a las dimensiones de lo narrativo, de acuerdo con la interpretación que hace de Genette (1980):

Podría centrarme en los movimientos retóricos que crean una "narración" o "exposición narrativa" particular; también podría centrarme en los sucesos y situación que se están describiendo, esto es, en las "historias" que se están contando, dado que los mismos acontecimientos pueden contarse de modos diferentes; y de hecho tiene que haber el "decir" real, sin el cual no habría exposición, y quizás tampoco una historia que contar (p. 32).

Conle se enfoca más en la producción del texto narrativo y diferencia el producto del relato, como una manifestación particular de una

1 Autodiegética se refiere al hecho de que el narrador narra en primera persona los acontecimientos de su propia vida. 
historia, que es presentado en un escenario específico. De nuevo, la narración (autobiográfica o no) construye la vida que se cuenta en la medida en que el sujeto hace la selección de los hechos a contar (la historia), así como el formato en que lo va a hacer (el producto) y actúa en un contexto en particular (el escenario).

Desde la intención educadora de su trabajo, Conle (2014) piensa que diferentes narrativas implican distintas dinámicas de aprendizaje, lo que es importante para entender los procesos educativos y su transformación. Supone una construcción propia de la realidad que afecta al modo en que los sujetos forman parte de ella y, en consecuencia, diseñan su actuación. Hay una intención transformadora inherente a los aprendizajes, de tal forma que el relato, más allá del contenido de lo que cuenta, es parte de la construcción de la subjetividad por medio de la cual nos enfrentamos al mundo.

\section{Las prácticas docentes y el relato de escuela}

De acuerdo con lo anterior, es posible revisar cómo se han pensado las prácticas docentes, de forma general, en la literatura científica. La profesión docente es una de las más reguladas en el marco de la sociedad actual (Knowles, 2004; Lortie, 1975; Zeichner \& Grant, 1981). Suele estar sometida a presiones continuas por parte de mútiples agentes, desde los políticos y los gestores educativos hasta las familias del alumnado y la opinión pública. Casi nunca se piensa la profesión docente en términos de lo que el propio profesorado vive como tal. Se generalizan, por ejemplo, los discursos acerca de las "buenas prácticas", que terminan por convertirse en nuevas prescripciones que incrementan el aparato normativo de la profesión (Fernández-Enguita, 1999; 2009). Esto se acompaña de un criterio moral que acentúa su acción reguladora. La práctica docente parece limitarse al cumplimiento de cierto tipo de reglas establecidas desde ámbitos diferentes.

Estas regulaciones constituyen, en mi opinión, la narrativa "oficial" de la profesión, que va construyendo un modo de hacer y de pensar que "obliga" la acción de los docentes en sus prácticas. Es un relato que configura una realidad desde el punto de vista de las agencias que lo elaboran. El problema se inicia cuando esta narrativa entra en conflicto con aquellas que elaboran los propios docentes y que son reducidas a la categoría de "conocimiento vulgar" y, por tanto, no válido para organizar la enseñanza (Cochram-Smith \& Lytle, 2002). En términos literarios, podría hablar de un "género menor" frente a la "gran literatura". El resultado suele ser la existencia de dos narrativas paralelas. La cotidianidad de la vida del docente queda alejada de las narrativas oficiales, lo que dificulta su transformación en un sentido que no sea el definido por la institución.

El punto de vista narrativo nos ofrece una forma de afrontar esta situación, sobre todo si atendemos al punto de vista constructivista y crítico. Clandinin (2013) afirma que vivimos lo que relatamos. En otras palabras, vivimos por medio del relato que hacemos de nuestra vida; por tanto, todo depende del relato que construimos. Como afirma tras su diálogo con Okri (1997), "si cambiamos las historias a través de las que vivimos es muy probable que cambiamos nuestras vidas" (p. 22). Los relatos nos transforman (Rivas, 2007; 2012b). En el encuentro con el otro mediante sus narraciones, se produce una modificación tanto de nuestra propia realidad como la del otro, lo que nos sitúa en el ámbito de las relaciones y sus implicaciones en la modificación de la realidad que construimos por medio de los relatos. De ahí que, si cambiamos el relato, transformamos nuestra realidad. Según Clandinin (2013): 
[...] pensar narrativamente acerca de la experiencia ilumina nuestra comprensión. Sus palabras [Okri, 1997] acerca de cambiar las historias a través de las que vivimos, cambiando quién somos, también dirigen mi atención a la ética relacional en relación al compromiso con la indagación narrativa ya que podemos cambiar no solo nuestras vidas y la de aquellos que viven en relación con nosotros, sino también las vidas de los participantes y de aquellos que viven en relación con ellos (pp. 22-23).

Podemos pensar que en el origen de los propios relatos personales está la intención de dar cuenta de la propia vida para poder transformarla. En este planteamiento encontramos un eje sobre el que gira el pensamiento narrativo. Cuando contamos nuestra historia, en definitiva, estamos hablando sobre experiencias, en especial, sobre la selección de acontecimientos con la que construimos nuestra experiencia. De hecho, como vengo planteando, no es posible acceder a la vivencia, a no ser por la reconstrucción que hacemos de ella; por tanto, no existe mientras no la relatemos. En otras palabras, entiendo la experiencia como el relato que hacemos de la vivencia. Benjamin (1991) expresa con elocuencia esta diferencia entre ambos conceptos, al referirse a los soldados que volvían de la guerra de trincheras de la Primera Guerra Mundial. Volvían enmudecidos, con lo que dejaban por fuera de los sucesos al resto de la población. Solo la posibilidad de contarlo hizo surgir la experiencia, más allá del cúmulo de libros escritos sobre el tema desde lo que Benjamin califica de "mentiras refutables" desde la voz de aquellos que lo vivieron.

\section{Lo individual y lo colectivo: el dilema moral}

En última instancia, la experiencia como relato de lo vivido es lo que construye nuestra vida, la realidad. Las prácticas docentes, por tanto, no son más que el relato que hacemos de ellas, para convertirlas en experiencia en la medida en que las compartimos. Así pues, la vivencia nunca es exclusivamente individual, sino que forma parte del relato colectivo con el que construimos nuestra realidad como comunidad. Frente al individualismo propio de la racionalidad liberal y positivista, entiendo que se trata de procesos históricos y sociales, por tanto, colectivos. Con base en autores como Harré (1984), pienso que la construcción de la realidad es el resultado de la relación dialéctica entre lo individual y lo colectivo, por un lado y lo público y privado, por otro, de modo que individuo y sociedad se constituyen mutuamente.

Concuerdo con Morin (1999) en que la identidad humana forma parte de una sociedad, a la vez que constituye la especie humana. El problema no solo está en relación con una ética individual, sino con una "ética para la humanidad". En definitiva, la solución no se halla en respuestas individuales a partir de las cuales cada quien resuelve sus propias prácticas, sino en prácticas que nos modifican colectivamente (o no) y que resultan de una responsabilidad compartida.

Llevado al terreno de la escuela, la experiencia que vivida construye un tipo de relaciones y unos modos de acción propios de lo que denomino "moral neoliberal" y que desarrollaré más adelante. En síntesis, se lleva a cabo el proyecto social, cultural y político que la ideología neoliberal plantea como escenario hegemónico para la sociedad actual. La configuración de las instituciones educativas establece unas condiciones particulares que ponen en juego dinámicas que construyen este tipo de prácticas. El contexto de producción del relato, en las palabras anteriores de Conle (2014), representa una de las dimensiones propias de construcción de la narrativa 
de las prácticas escolares, en una perspectiva que califico de histórica y colectiva.

En el caso de las escuelas, este espacio no es ajeno a su propia constitución como institución de la modernidad (Díaz \& Rivas, 2007). Si bien ha ido evolucionando fenotípicamente en sus 250 años de historia, se puede hablar de un genealogía particular que la ha configurado en lo que es hoy, originado en el proyecto que representaba. Hablo de un nuevo marco caracterizado por un pensamiento racional con el que pretendía alcanzar un estado de emancipación de la especie humana, de las servidumbres propias del trabajo manual y de la relación con la naturaleza. En la medida en que la modernidad es un proyecto social, cultural y político del colectivo que se convierte en hegemónico en virtud de las revoluciones burguesas, la escuela viene marcada por su afán colonizador y de construcción de una nueva moral ciudadana acorde con la nueva realidad. La escuela adquiere el encargo de transmitir la nueva narrativa emergente, con carácter universal. Según Carnoy (1977), los sistemas educativos que surgen como consecuencia del nuevo orden representan una forma de colonización de las clases trabajadoras, para convertirlas en sujetos aptos para el Estado moderno y la sociedad industrial.

En coherencia con la lógica modernista, se instaura un cuerpo profesional encargado de llevar a cabo esta finalidad a la cual se le dota de unas características particulares. El hecho de que la educación esté encuadrada dentro de las obligaciones de los nuevos Estados modernos genera unas condiciones propias del trabajo docente, dependiente del cuerpo político y, en especial, de la dinámica administrativa y burocrática que los caracteriza. Las prácticas están prediseñadas por un mandato institucional, al tiempo que se las quiere conferir de un carácter profesional y científico. Este último sirve de justificación y legitimación para dejar que dicha exigencia institucional actúe con garantías. El surgimiento de los estudios de magisterio (Rivas, 2004) brinda un modelo formativo profesional, que le confiere una autoridad técnica con la que legitima su tarea.

De este modo, se cierra la coherencia interna de la propuesta, lo que genera una línea continua que inicia en la escuela - con el rol de alumno-, continúa con la formación en la universidad —que actúa con una lógica isomórfica con la escolar-y se cierra en la inserción en la misma escuela con el rol docente. Las prácticas docentes no se transforman, pero sí se legitiman.

Resulta relevante la metáfora que nos proponen Pineau, Dussel y Caruso (2001), acerca de la escuela como "máquina de educar". Existe un aparato técnico diseñado para actuar en cualquier espacio y lugar, de acuerdo con el ideal racionalista que lo sustenta. Este principio ha estado y está muy arraigado en el funcionamiento de la escuela. Su carácter prescriptivo, la idea de la educación como conjunto de técnicas pedagógicas y el rasgo moral con el que se adorna, entre otros, son componentes sustanciales que definen el "cómo" de la escuela.

El neoliberalismo, con su paradójica tensión entre emancipación y regulación ${ }^{2}$, es legítimo heredero de esta atmósfera, por lo que establece pautas precisas de actuación del docente, acordes con la narrativa histórica que se ha ido construyendo colectivamente en los sistemas educativos. Quizá se produce un cambio importante respecto a etapas anteriores; más que criterios científicos definidos con planteamientos pedagógicos, ahora se enfatiza en el diseño moral que representa: el discurso está centrado en la tarea, sobre todo en su efectividad en cuanto al mantenimiento del orden (no tanto al contenido del aprendizaje), dentro de un sistema credencialista legitimado desde agencias externas.

A continuación presento una breve presentación de la investigación sobre la que se construye este trabajo, para después desarrollar alguno de los ejes fundamentales de la presencia de la moral neoliberal en la escuela.

\section{La investigación}

La investigación analiza la identidad profesional que se construye en los estudios de profesorado de primaria e infantil en dos momentos: el primero, que intenta reconstruir la experiencia escolar del alumnado del grado de Primaria cuando empieza su primer año en la universidad y el segundo, en el que se examina la primera experiencia profesional de este alumnado, una vez inicia su carrera profesional.

A efectos de contextualizar la lectura, las preguntas que mueven la investigación son: ¿Qué vínculo existe entre la experiencia profesional como docente y la experiencia durante sus años escolares? ¿Hay coherencia entre el conocimiento adquirido en estas experiencias tempranas y el que luego se pone en juego como profesionales? El relato personal, tanto del alumnado que entra a primero del grado de Primaria en la universidad como del profesorado novel en ejercicio, es el eje del análisis. Hay una diferencia en la elaboración de estas narraciones: en el primer caso se trata de un texto escrito por los propios sujetos,

2 Según explico en Díaz y Rivas (2007) y en Rivas (2012a), el liberalismo, como parte del proyecto de modernidad, plantea como uno de sus fines la emancipación del sujeto frente a los sufrimientos de la naturaleza, si bien al mismo tiempo, se enfrenta a una espiral de creciente regulación de la vida pública para sostener la inestabilidad inherente a la sociedad de mercado. Ello confronta al neoliberalismo con lo que considero una de sus grandes falacias. 
mientras en el segundo se construye desde los relatos orales del profesorado, que luego fueron escritos por ellos mismos.

Estos documentos fueron trabajados colectivamente por los autores, lo cual permitió tener en cuenta sus puntos de vista y sus interpretaciones a la hora de iniciar el proceso de análisis. Más adelante, el grupo de investigación procedió a identificar los ejes problematizadores que facilitan la búsqueda de sentido de la experiencia escolar y valorarlo de cara a los procesos de formación de profesorado. Estos ejes fueron discutidos en ambos escenarios, con el fin de ampliar su marco de explicación. De estos relatos surgieron dos esquemas de interpretación.

En primer lugar, los relatos del estudiantado llevaron a diferenciar cuatro ejes:

- Idealización del trabajo docente.

- Rutinas en la experiencia escolar.

- Evaluación como práctica continuada en la escuela.

- Autoridad, disciplina y orden.

Estos núcleos muestran un sistema de comprensión de la profesión que enfatiza las tareas y la autoridad del docente, gestionado mediante procesos de evaluación. Esto supone, desde mi punto de vista, una ruptura con la lógica redentorista (Popkewitz, 1998) de la educación, muy presente en otros momentos históricos, que plantea su lugar preeminente para el cambio de la sociedad. En estos momentos se remarca más en la autoridad y en la tarea que en la capacidad de la educación para cambiar el mundo.

En segundo lugar, el trabajo con el profesorado novel se categoriza en otros cuatro ejes:

- Relaciones complejas entre la formación y la profesión.

- Imaginarios ideológicos que se construyen en esta relación.

- Presencia de experiencias relevantes en el inicio de la profesión.

- Condiciones profesionales en las que actúan y que suponen un marco complejo.

El profesorado construye sus prácticas profesionales a partir de cómo va resolviendo estas cuatro dimensiones en su cotidianidad, dentro de un marco comunitario y colectivo que aporta una historia y unas tradiciones propias.

En el momento actual, estos dos conjuntos de dimensiones van traduciendo las condiciones sociales, políticas, culturales y económicas en las que se constituyen, de manera que las propuestas hegemónicas que se desarrollan en estos ámbitos empiezan a for- mar parte de la vida escolar mediante la acción de los sujetos y el tipo de construcción social que elaboran en el conjunto de su experiencia. A pesar de que la escuela conforma un marco institucional definido, no queda indemne a la acción de los sujetos que aportan las contingencias socioculturales. Asimismo, la política educativa y su desarrollo administrativo generan condiciones propias que aportan un contenido a este entramado complejo que llamamos escuela.

A continuación, desarrollo el contenido de estas vivencias escolares con base en dos núcleos del relato de escuela que se está produciendo en el contexto neoliberal. De una parte, lo que en la investigación denomino "conocimiento sedimental", ese que se construye desde la experiencia cotidiana y que permanece en el lecho de la experiencia escolar y, de otra, lo que nombro "moral neoliberal", en cuanto al modo como se articulan las prácticas escolares en estas condiciones sociales, políticas, culturales y económicas.

\section{Los relatos de la práctica escolar}

Como ya he definido, la práctica docente es una construcción que tiene lugar con base en procesos históricos, institucionales, culturales, educativos y también personales. Si bien podemos hacer la analogía de un ADN institucional en la escuela, que ofrece un marco determinado de posibilidades (quizá más cerrado de lo que a muchos nos gustaría), el devenir social lo modula fenotípicamente y lo hace viable en las distintas coyunturas sociohistóricas. En este sentido, el actual marco neoliberal y la colonización que está haciendo de los espacios públicos y privados se patenta en las diversas facetas de la vida escolar, acompañado, en el caso español, de un halo conservador que le sirve de legitimación moral.

Esto se puede corroborar en el modo como está cambiando el relato de escuela en los sujetos de la investigación. El significado de los procesos educativos, aun con los principios básicos de su constitución, está modificándose por la incursión de esta nueva "moral neoliberal"; entran con fuerza modelos de actuación que no creo equivocado calificar de mercantilizados 0 , al menos, instrumentalizados desde ese discurso.

En este contexto, el conocimiento sedimental y la moral neoliberal imperante son los dos ejes que emergen en este análisis.

\section{El conocimiento sedimental}

Estoy acuñando este concepto para analizar y comprender el conocimiento que se construye en el paso por la escuela como estudiante y el tipo de experiencia vivida acerca de qué es y cómo funciona la profesión docente (Rivas, 2014). La experiencia escolar 
elabora un conocimiento profesional que se va sedimentando a lo largo de su paso por la escuela. Según el DRAE, el sedimento es la "materia que se deposita en el fondo por su mayor gravedad. En sentido figurado tiene que ver con aquello que permanece y que trasciende a las coyunturas particulares" y constituye la base sobre la que se construye la futura profesión docente, tanto por lo que se acepta tácitamente y forma parte de lo deseable en la profesión como por aquello que es objeto de revisión y crítica. Este conocimiento puede entrar en conflicto o diferir de los postulados teóricos y conceptuales que ofrece el conocimiento pedagógico elaborado, que a menudo se plantea en formato prescriptivo sin tener en cuenta el "suelo" que lo sustenta. Esto puede generar una reacción entre ambas, al suscitar nuevas posibilidades o bien crear otra capa sobre el sedimento, mucho más endeble y transitoria de lo que nos gustaría pensar desde el ámbito académico. A menudo, ambas capas coexisten en parcelas distintas del cauce y forman parte de sistemas de legitimación diferentes.

Kush (1976) define esta situación como "conocimiento situado", esto es, aquel que está ligado a un momento y lugar determinados y que depende de las condiciones culturales y materiales concretas de un marco social. Es un "estar-siendo" que se traduce en la "posibilidad de ser" en cada escenario particular. El conjunto de experiencias que presentan los relatos saca a la luz este conocimiento.

Desarrollo una serie de ejes sobre los que se erige y que muestran un escenario peculiar, no exento de complejidad. Estos núcleos, desarrollados en otros trabajos (Rivas, 2014; Rivas \& Leite, 2013; Rivas, Pérez, Leite \& Quijano, 2013), son:

- La enseñanza se entiende como una estructura de tareas.

- El aprendizaje consiste en la adquisición de los contenidos del currículum.

- El orden es necesario para la realización de las tareas.

- Solo es válido aquello que se evalúa.

- La autoridad del docente se establece en relación con el rol que desempeña.

- La escuela es el espacio profesional del docente.

- Existen teorías innatistas y genetistas en la justificación de los procesos escolares.

La mayoría de estos ejes está orientada hacia un modelo social de marcado tipo gerencialista, en el que importa la actividad en sí misma, el control, la disciplina, la jerarquización y la segregación de roles, entre otras cosas. Resulta una visión bastante alejada del idealismo que se presupone en el alumnado de educación y sobre el que, en buena parte, se sustentan las expectativas del profesorado en la universidad.

Un alumno muestra con claridad cómo percibe este proceso de adquisición de pautas profesionales que generan procesos identitarios sobre los que construir su profesión:

Tuve un profesor de Primaria que nos impartía todas las asignaturas, excepto Educación Física y Religión, durante tercero y cuarto curso y me ha dejado una huella para toda la vida. Él siempre nos contaba sus anécdotas y sus problemas de cuando era joven, con la intención de interactuar con nosotros y tener más apego hacia él, y también para que nos sirva para conocer que la vida no es tan bonita como cree un adolescente. Nos exigía mucho y suspendía casi a toda la clase, y nos decía: "Luego os alegraréis cuando lleguéis a Bachillerato y tengáis nivel", y efectivamente, me dí 
cuenta cuando ya no nos daba clase, y son muchos de sus consejos los que me han hecho reflexionar (alumno A, comunicación personal, 2010).

El énfasis en la tarea y en el currículum y el modo como este se convertía en perspectiva, dentro de un marco de relación, establecen un sistema de pensamiento para el futuro profesor, del que "se dio cuenta" más adelante. Tal relación, que en este caso es afectiva y cercana, con dosis de autoridad y control, en otros casos es narrada desde miradas menos complacientes.

Luego, este conocimiento es renovado desde otra perspectiva por el profesorado novel en ejercicio. Si revisamos expresiones del tipo "tienes que buscarte la vida para enfrentarte a la tarea docente", "te dan la peor clase", "los niños no paraban de hablar; qué hago con estos niños", se adivina un sedimento de este conocimiento. La preocupación por la tarea, la necesidad de control, el trabajo individual y la necesidad de "hacer cosas" muestran un trabajo muy centrado en la necesidad diaria y cotidiana, caracterizada por la inmediatez, en el que lo importante es mantener una cierta actividad. Esto supone, a menudo, una pérdida de las finalidades generales del trabajo docente, de una visión social del mismo e, incluso, un menoscabo del valor de los principios educativos desarrollados en la universidad. La inutilidad de los años de formación inicial, salvo el paso por el practicum, parece ser, desde sus relatos, una constante en la profesión. Esto redunda en una visión a corto plazo y, en consecuencia, una mayor dependencia de los materiales editados y los libros de texto, que se convierten en un colchón de seguridad.

La visión gerencialista de la profesión, propia de una sociedad neoliberal, se hace evidente en este conocimiento y en el modo como se traduce en prácticas docentes. El tradicional discurso redentorista y vocacional queda subsumido, en parte, por un visión pragmática, activista y eficaz. Estaría muy en consonancia con lo que Sennett (2012) denomina "pensamiento cortoplacista", según el cual todo se reduce a resolver el momento concreto, el día a día y la inmediatez de la relación pedagógica. En este contexto, las grandes finalidades y los proyectos educativos quedan relegados $\mathrm{O}$, al menos, mediatizados por este conocimiento, muy marcado por las condiciones de la experiencia escolar, que vendrían a caracterizarse por lo que vengo denominando "moral neoliberal". En otras palabras, cómo el proyecto social, cultural y político que representa el neoliberalismo se concreta en una serie de prácticas particulares que hace realidad este proyecto. No solo estoy hablando de una ideología, de un desarrollo teórico o filosófico, sino de prácticas sociales (profesionales en este caso).

\section{Características de la moral neoliberal}

La sociedad neoliberal presenta un conjunto de rasgos que la definen frente a otros proyectos sociales. Estas vendrían a concretar y "operativizar" el proyecto que representa. Repaso aquellas que tienen más incidencia sobre el modelo educativo y luego valoraré cómo estas características forman parte de las narraciones del alumnado y del profesorado novel. Entiendo que una de las cuestiones más graves que se presentan en el sistema educativo español es que está siendo permeable a la acción de la sociedad mercantilizada neoliberal, por lo que incorpora prácticas y actos congruentes con esta propuesta.

Cabe pensar que el origen modernista de la propia escuela actual tiene que ver con el nacimiento de la sociedad industrial y el triunfo del liberalismo económico. Debido a ello, las instituciones escolares han trabajado 
de acuerdo con el desarrollo de esta propuesta, con más peso del racionalismo. Si pensamos en el pacto liberal-racional que da origen a los Estados modernos, siempre se ha trabajado en el mismo sentido. El cambio sustancial de los últimos años es que el utilitarismo y "practicismo" neoliberal ha ido desplazando los valores del racionalismo y ha quedado como hegemónico.

En los últimos años, en España se ha dado una vuelta atrás en materia educativa, al tiempo que se han implementado nuevas prácticas que suponen el triunfo de las posiciones propias del neoliberalismo más duro (control, eficacia, homogeneización, etc.) (Rivas, 2013). Los discursos educativos basados en la transformación social, la innovación educativa o la mejora de la sociedad están dejando paso a aquellos otros que hablan de la tarea, de la profesionalidad o del currículum preestablecido. La acción de los docentes se ha transformando en un proceso técnico, basado en la aplicación de estrategias elaboradas fuera de su realidad institucional. Esta pérdida de autonomía genera un cambio en el discurso educativo que está empezando a formar parte de la ideología profesional de los docentes.

En términos generales, esto es lo que denomino "sociedad gerencialista". La principal característica del neoliberalismo es la condición de gestor de los diferentes actores educativos: docentes y directivos se están convirtiendo en gestores de libros de texto, de currículos oficiales, de disposiciones y normativas establecidas, etc. "El primer problema de la profesión es mantener el orden para poder enseñar", dicen los docentes y corroboran los estudiantes que incian sus estudios profesionales.

Entonces, ¿qué significa enseñar? ¿Qué sentido le damos al sujeto, al conocimiento, a la actividad escolar? Hay nuevas construcciones que ya estaban presentes en la "genética institucional" de la escuela; se han actualizado o, en su caso, se han exacerbado. La cultura del control forma parte importante de esta moral, fruto de la obsesión por el orden. Mientras todo esté controlado y no se rompa la lógica de la acción, no hay problema. La pérdida de las finalidades es notoria: qué se aprende, por qué y para qué, es un referente lejano que, de cuando en cuando, nos sirve para valorar los desacuerdos más patentes. La inmediatez de la respuesta en la escuela, sin duda, es un factor que contribuye a construir esta idea. "Cuando lleves unos años" es una expresión cotidiana, evocada por el profesorado novel para recordar su inserción profesional, como aviso a navegantes de la inutilidad de la formación recibida. Es el mismo caso de un docente que comenta que "la programación no sirve para nada". Se hace referencia a unas condiciones de trabajo determinadas (que contribuyen a la generación de esta situación) que nos habla de la dificultad de mantener unos criterios reflexivos. La cotidianidad se come la tarea docente y contribuye al desarrollo de esta moral.

Este comportamiento conduce a otro elemento que configura esta moral: el "como si", es decir, la cultura de la apariencia como forma de funcionamiento de la escuela. La cultura escolar, de alguna forma, se construye sobre una ficción o un conjunto de ellas. La más relevante es la ficción del aprendizaje: se evalúa como si la calificación fuera reflejo del aprendizaje del alumnado, aunque también existen la apariencia de control y ausencia de conflictos, la ficción de la igualdad curricular frente a las diferencias de clase y la ficción de la homogeneidad por grupos de edad. En definitiva, el sistema se sostiene sobre unos supuestos que se correlacionan con los valores del proyecto neoliberal de la sociedad, sostenido por un sistema económico-financiero que lo legitima por la vía de la hegemonía política y social y no por la de la justicia social y el respeto a los derechos humanos.

Me parece relevante reseñar cómo las prácticas escolares están construyéndose desde esta perspectiva y estos valores. Entiendo que la escuela no sabe o no puede hacer frente a esta propuesta, salvo desde una acción intencional y explícita, que recupere lo significativo frente a lo aparente y las finalidades frente al activismo. Es interesante que el profesorado y el alumnado que relatan su paso por experiencias educativas diferentes se sienten impelidos a otro tipo de actuación, aun dentro de la dificultad de la estructura establecida.

Buena parte del alumnado resalta experiencias relevantes con profesorado que tuvo en la escuela, así como algunas propuestas alternativas; estas últimas a menudo tienen lugar fuera de la propia escuela. Esto da la pauta de que es posible otra moral, en la medida en que vivencias educativas diferentes van formando parte de la dimensión más visible de la escuela. Por ejemplo, un alumno resalta en su relato el impacto de un profesor que "se salía de la norma":

El maestro intentaba unirnos como grupo. Con él cada día en la escuela era una oportunidad para aprender algo nuevo, todos sabíamos que en cualquier momento nos podía sorprender con una experiencia totalmente novedosa. Su implicación con los alumnos no se limitaba a lo concerniente al aula, sino que iba más allá, se preocupaba por todos y trató de enseñarnos de manera activa (alumno B, comunicación personal, 2010).

El problema está en saber si la formación docente puede potenciar estas experiencias y generar procesos de cambio y transformación en el discurso hegemónico o si, en cambio, actúa como cortacircuitos de las mismas y anula su potencial. El tipo de vivencia 
organizativa de las facultades de educación no necesariamente contribuye a proponer otro tipo de experiencias significativas ni a reforzar estos acontecimientos relevantes con los que llega el alumnado. No se trata de un cambio de discurso, sino de un cambio de vivencias tanto en la escuela como en las instituciones de formación.

\section{Conclusión}

Como toda realidad, la práctica docente es una construcción colectiva, fruto del relato que los distintos sujetos, en diferentes contextos, construimos histórica y socialmente. Por tanto, las narraciones que ofrecen los docentes y estudiantes de la investigación revelan qué tipo de realidad se está construyendo en los escenarios sociales, educativos y políticos actuales. Además, evidencian cuáles son estos escenarios y qué representan. Es más, también muestran hacia dónde nos conducen.

Entiendo que, más allá de este relato hegemónico, se están construyendo otros, producto de diferentes proyectos sociales, educativos y políticos, que actúan contrahegemónicamente, al desarrollar otras prácticas profesionales que no dejan de ser sociales y políticas. El trabajo narrativo permite visibilizar estas otras construcciones de la realidad y, a la vez, cuestionar el relato vigente, como del compromiso con proyectos de sociedad diferentes.

He intentado mostrar cómo tiene lugar este proceso que señala el contenido ideológico y epistemológico presente en las narrativas neoliberales que están colonizando los espacios institucionales. Tal contenido no es solo un discurso, sino que, en la medida en que los sujetos se apropian de él, empieza a formar parte de sus prácticas y modos de hacer en los diversos ámbitos de su vida. Por tanto, estoy hablando de un relato que construye la realidad del sujeto y de su entorno en una relación interconstituyente.

La moral neoliberal es más que una propuesta de vida, pues supone un conjunto de prácticas incorporadas a la vida individual y colectiva que orientan la acción.

El análisis narrativo permite descubrir los otros relatos que pueden estar construyendo realidades alternativas que orienten otro tipo de acciones. En este caso, el hecho de hacer visible el que se presenta como hegemónico promueve compromisos de tipo social, cultural, político o educativo, a favor de la transformación y la mejora social. Churchill (2008) lo define como preguntarse sobre uno mismo y esto nos induce a preguntarnos sobre "nosotros" mismos. Por ello, todo relato es personal y biográfico, construido históricamente, a partir de las diferentes experiencias que vivimos. Lo que importa más que el actor (el yo que vive la experiencia) es el narrador (el yo que la cuenta) en un momento dado de su trayectoria. Hacer públicos estos relatos en un contexto de libertad propicia condiciones para su transformación.

Concluyo con una referencia literaria de Saramago (2012). No existen ejemplos de vida; más bien se trata de nuevos retos sobre los cuales pensar y hacer.

—¿Qué piensa hacer, Abel?

El muchacho se levantó lentamente y se dirigió hacia Silvestre. A dos pasos, le respondió:

-Algo muy simple: vivir. Salgo de su casa más seguro de lo que estaba cuando entré. No porque me sirva el camino que me ha apuntado, sino porque me hizo pensar en la necesidad de encontrar el mío propio. Será cuestión de tiempo (p. 415). 


\section{Sobre el autor}

José Ignacio Rivas-Flores es catedrático de Didáctica y Organización Escolar en la Universidad de Málaga (España) y es responsable del grupo de investigación ProCie (Profesorado, Cultura e Institución Educativa). Interesado en la formación del profesorado, la investigación biográfico-narrativa y los procesos institucionales en los sistemas educativos modernos.

\section{Referencias}

Benjamin, W. (1991). El narrador. Madrid: Taurus.

Carnoy, M. (1977). La educación como imperialismo cultural. México D. F.: Siglo XXI.

Clandinin, D. (2013). Engaging in Narrative Inquiry. Walnut Creek: Left Coast Press.

Cochram-Smith, M., \& Lytle, S. (2002). Dentro/fuera. Enseñantes que investigan. Madrid: Akal.

Conle, C. (2014). Anatomía de un currículo narrativo. En Rivas, J. I., Leite, A., \& Prados, E. (eds.). Profesorado, escuela y diversidad. La realidad educativa desde una mirada narrativa. Archidona: Aljibe.

Churchill, A. H. (2008). The Emocional Journey into Critical Discourses: Appreciating the Challenge. En Churchill, A. (ed.). Rocking your World. The Emocional into Critical Discourses. Rotterdam: Sense.

Díaz, I., \& Rivas, J. I. (2007). Un nuevo modelo de mujeres africanas: el proyecto educativo colonial en el África Occidental francesa. Madrid: CSIC.

Fernández-Enguita, M. (1999). La profesión docente y la comunidad escolar: crónica de un descubrimiento. (3a ed.). Madrid: Ediciones Morata.

Fernández-Enguita, M. (2009). Educar en tiempos inciertos. (3a ed.). Madrid: Ediciones Morata.

García-Márquez, G. (2002). Vivir para contarla. Barcelona: Mondadori.

Genette, G. (1980). Narrative Discourse. Oxford: Basil Blackwell.

Harré, R. (1984). Personal Being. A Theory for Individual Psychology. Cambridge: Harvard University Press.

Knowles, J. (2004). Modelos para la comprensión de las biografías del profesorado en formación y en sus primeros años de docencia. Ilustraciones a partir de estudios de caso. En Goodson, I. (ed.). Historias de vida del profesorado. Barcelona: Octaedro.

Kush, R. (1976). Geocultura del hombre americano. Buenos Aires: Fernando García Cambeiro.

Lejeune, P. (1994). El pacto autobiográfico y otros estudios. Madrid: Megazul-Endymion D. L.

Lortie, D. (1975). Schoolteacher. Chicago: University of Chicago Press.

Morin, E. (1999). Los siete saberes necesarios para la educación. París: Unesco.

Okri, B. (1997). A Way of Being Free. Londres: Phoenix House.

Pineau, P., Dussel, I., \& Caruso, M. (2001). La escuela como máquina de educar. Buenos Aires: Paidós.

Popkewitz, T. (1998). Los discursos redentores de las Ciencias de la Educación. Sevilla: MCEP.

Rivas, J. I. (2004). ¿Cómo enseñar a los que enseñan? Introducción a la evolución de la deformación y formación del profesorado. En Guerrero, J. F., \& Pérez-Galán, R. (eds.). La pizarra mágica. Una visión diferente de la historia de la educación. Archidona: Aljibe.

Rivas, J. I. (2007). Vida, experiencia y educación. La biografía como estrategia de conocimiento. En Sverdlick, I. (comp.). La investigación 
educativa. Una herramienta de conocimiento y de acción. Buenos Aires: Novedades Educativas.

Rivas, J. I. (2012a). Regulación vs. participación: sobrevivir democráticamente en tiempos difíciles. Diálogos. Educación y formación de personas adultas, 3(71-72), 57-70.

Rivas, J. I. (2012b). Sujeto, diálogo, experiencia: el compromiso del encuentro. En Rivas, J. I., Hernández, F., Sancho, J. M., \& Núñez, C. (eds.). Historias de vida en educación. Sujeto, diálogo, experiencia. Recuperado de http://hdl.handle.net/2445/32345

Rivas, J. I. (2013). El paso del cangrejo en el baile de las reformas: "lomceando" la educación pública. Ateneo del Nuevo Siglo (17), 54-57.

Rivas, J. I. (2014). Nuevas identidades en la formación del profesorado: la voz del alumnado. International Journal of Development and Educational Psychology, 7(1), 487-494.

Rivas, J. I., \& Leite, A. (2013). Aprender la profesión desde el pupitre. Cuadernos de Pedagogía (436), 34-37.

Rivas, J. I., Pérez, M., Leite, A. E., \& Quijano, R. (septiembre de 2013). Previous Pedagogical Beliefs of Freshmen of Teacher Education. Conferencia presentada en European Conference on Educational Research [ECER], Estambul, European Educational Research Association y Bahçeşehir University in Istanbul.

Saramago, J. (2012). Claraboya. Madrid: Anagrama.

Sennett, R. (2012). Juntos. Rituales, placeres y política de cooperación. Barcelona: Anagrama.

Zeichner, L., \& Grant, C. (1981). Biography and Social Structure in the Socialization of Student Teacher. A Re-Examination of the Pupil Control Ideologies of Students Techers. Journal of Education for Teaching, 7(3), 298-314 